\title{
Impact of betatron collimation losses in the High-Energy Large Hadron Collider
}

\author{
M. Varasteh $\odot,{ }^{* \dagger}$ R. Bruce, F. Cerutti®, M. Crouch, and F. Zimmermann \\ European Organization for Nuclear Research (CERN), \\ Esplanade des Particules 1, 1211 Geneva, Switzerland
}

(Received 11 October 2020; accepted 5 March 2021; published 1 April 2021)

\begin{abstract}
In response to recommendations in the 2013 update of the European Strategy for Particle Physics, a conceptual design effort for an energy upgrade of the Large Hadron Collider (LHC) at CERN, the so-called high-energy LHC (HE-LHC), was launched as part of the Future Circular Collider study. The HE-LHC machine, which is meant to use $16 \mathrm{~T}$ magnet technologies in the existing LHC tunnel, would provide proton collisions at a center-of-mass energy of $27 \mathrm{TeV}(\sim 2 \times$ LHC $)$ with a total stored energy of $1.34 \mathrm{GJ}$ $(\sim 4 \times$ LHC) per beam. By adapting the LHC collimation system, a first layout of the HE-LHC's betatron cleaning insertion was conceived, with the requirement to sustain—for at least 10 seconds—-the impact of about $1.86 \mathrm{MW}$, corresponding to a beam lifetime of 12 minutes, without inducing any magnet quench nor any damage to other accelerator components. In this article, we evaluate the power deposition on the collimation insertion for proton beam operation in the HE-LHC machine at top energy, by means of particle tracking and interaction calculations. The beam loss effects on the warm elements as well as on the superconducting dispersion suppressor magnets are assessed through a three-step simulation approach. In particular, for the proposed future high-energy LHC, we demonstrate the necessity of adding local collimators in the dispersion suppressor, and we uncover the harmful consequences of a potential removal of the beam line "dogleg" in the collimation insertion.
\end{abstract}

DOI: 10.1103/PhysRevAccelBeams.24.041601

\section{INTRODUCTION}

With the aim of proposing designs for a post-LHC particle accelerator, the Future Circular Collider (FCC) study developed and evaluated three accelerator options, which are documented in a four-volume FCC conceptual design report: a $100 \mathrm{~km}$ circumference hadron collider (FCC-hh) carrying a total energy of about $8500 \mathrm{MJ}(\sim 20$ times the LHC), a highest-luminosity high-energy lepton collider (FCC-ee), and an energy upgrade of the LHC based on FCC-hh technology to increase the center-of-mass energy by a factor of 2 (HE-LHC) [1-4].

The HE-LHC design assumes the usage of the FCC-hh $16 \mathrm{~T}$ magnet technology inside the existing LHC tunnel infrastructure in order to provide $p p$ collisions at a centerof-mass energy of $27 \mathrm{TeV}$ [3]. Main parameters are outlined in Table I. At a nominal beam intensity of $6.18 \times 10^{14}$, the total energy stored in each beam would

\footnotetext{
*m.varasteh.a@gmail.com

'Present address: Patrick G Johnston Centre for Cancer Research, Queen's University Belfast, Belfast, United Kingdom.

Published by the American Physical Society under the terms of the Creative Commons Attribution 4.0 International license. Further distribution of this work must maintain attribution to the author(s) and the published article's title, journal citation, and DOI.
}

be equal to $1.34 \mathrm{GJ}$, which is about a factor of 4 beyond the LHC design value [5]. As a design criterion we specify that the HE-LHC collimation system is required to cope with a temporary drop of the beam lifetime (BLT) down to 12 minutes, corresponding to an instantaneous beam loss power of $1.86 \mathrm{MW}$. This loss power has to be sustained over ten seconds without any superconducting (SC) magnet quench. This requirement is identical to what was used in the designs of LHC [6] and HL-LHC [7]. LHC operational experience has shown that such short beam lifetimes occurred only on very few occasions [8]. This is thus a conservative assumption, which we keep for HE-LHC in order to stay on the safe side.

Even a tiny fraction of such beam losses could lead a superconducting (SC) magnet to quench. In order to properly dispose of the lost beam particles, an ultraefficient intercepting mechanism is required to extract the beam halo particles and absorb part of the induced shower, while redirecting the rest towards less sensitive equipment. In the HE-LHC design, the insertion region IR7 remains dedicated to betatron cleaning and the insertion region IR3 to off-momentum cleaning. The stored energy increase, together with the stringent constraints imposed by the geometry of the existing $27 \mathrm{~km}$ tunnel, poses a significant challenge to the design of the collimation system for the HE-LHC. 
TABLE I. Main design parameters for HE-LHC [3], HL-LHC [7] and LHC [5]. The luminosity refers to a leveled value for HL-LHC and to the peak for HE-LHC and LHC.

\begin{tabular}{lcccc}
\hline \hline Parameters & Unit & HE-LHC & (HL)-LHC & Ratio \\
\hline Center-of-mass energy & $\mathrm{TeV}$ & 27 & 14 & 1.93 \\
Arc dipole field & $\mathrm{T}$ & 16 & 8.33 & 1.92 \\
Beam lifetime & $\mathrm{min}$ & 12 & 12 & 1.00 \\
Number of bunches/beam & & 2808 & $(2736) 2808$ & $(1.03) 1.00$ \\
Bunch intensity $\left[\times 10^{11}\right]$ & & 2.2 & $(2.2) 1.15$ & $(1.00) 1.91$ \\
Beam intensity $\left[\times 10^{14}\right]$ & & 6.18 & $(6.02) 3.23$ & $(1.03) 1.91$ \\
Proton loss rate $\left[\times 10^{11}\right]$ & $\mathrm{p} / \mathrm{s}$ & 8.58 & $(8.36) 4.49$ & $(1.03) 1.91$ \\
Beam loss power & $\mathrm{MW}$ & 1.86 & $(0.938) 0.503$ & $(1.98) 3.70$ \\
Stored energy/beam & $\mathrm{MJ}$ & 1336.2 & $(675.1) 362.2$ & $(1.98) 3.69$ \\
Normalised transverse emittance & $\mu \mathrm{m}$ & 2.5 & $(2.5) 3.75$ & $(1.00) 0.67$ \\
Luminosity $\left[\times 10^{34}\right]$ & $\mathrm{cm}^{-2} \mathrm{~s}^{-1}$ & 16 & $(5) 1$ & $(3.20) 16.0$ \\
\hline \hline
\end{tabular}

An accurate prediction of the collimation system's cleaning efficiency and its global performance is required to make sure that the SC magnets are sufficiently protected, and that the collimators themselves and the nearby equipment will not suffer any damage. In this article we focus on betatron losses, which are deemed most critical. Studies for the LHC [9], HL-LHC [10,11], and FCC-hh [12] have shown that, in each of these machines, the dispersion suppressor (DS) downstream of IR7 is the ring location with the highest losses on SC magnets caused by leakage from the collimation system. The same situation is also confirmed for HE-LHC by the tracking studies in Sec. III A. Therefore, the detailed energy deposition studies in this article focus mainly on IR7, while other loss locations around the ring are considered less critical.

Previous studies [9,13-16] established that the SixTrack $[17,18]$ particle tracking code and the FLUKA [19-21] particle interaction code represent a powerful combined simulation tool to study the collimation performance. In the present work we have evaluated the betatron losses and related energy deposition by adopting this wellbenchmarked simulation chain. Assuming machine operation at the nominal top beam energy $(13.5 \mathrm{TeV})$, here we present the results of the particle shower calculations. The power deposition in the warm elements within the long straight section (LSS) as well as in the dispersion suppressor (DS) SC magnets is reported in detail. More notably, a comprehensive assessment of scenarios contemplating the absence and presence of the DS collimators as well as the LSS dogleg is demonstrated. Results are normalized to the design beam loss scenario and compared to those for the LHC.

\section{BETATRON CLEANING INSERTION OF THE HE-LHC MACHINE}

In the HE-LHC baseline layout, a three-stage cleaning hierarchy is foreseen for the IR7 insertion region. The collimation system design is based on those of the LHC [22-26] and high-luminosity LHC (HL-LHC) [7,10,11,27], but the lattice layout is not identical, with the LSS about $34 \mathrm{~m}$ longer. The general layout and optics are discussed in [3]. A further refined optics with improved geometry matching for the insertion of DS collimators is reported in Ref. [28]. The HE-LHC arcs are organized in FODO cells (periodic structure of focusing quadrupole, drift space, defocusing quadrupole and drift space). For convenience, the elements in the betatron cleaning insertion are also grouped in so-called cells (see Fig. 1), numbered according to the quadrupoles, although the optics and lattice are

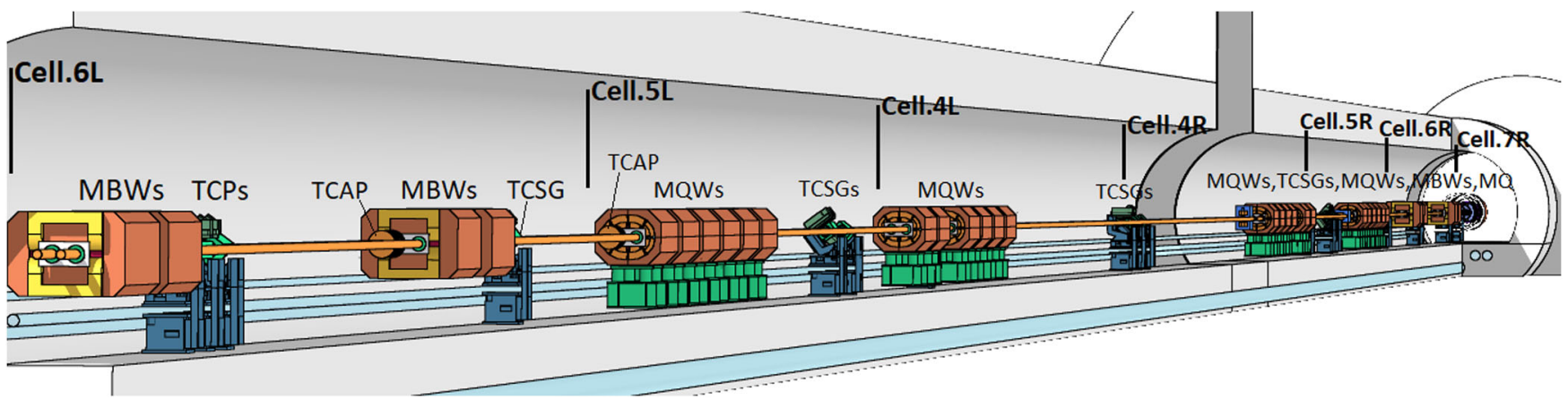

FIG. 1. FLUKA geometry model of the HE-LHC betatron cleaning insertion (warm section). The first object on the left is the MBW.D6L7 warm dipole, located $232 \mathrm{~m}$ upstream of the LSS center (IP7). 
different from the arc. The collimation system described in [3] includes three primary collimators called TCP (vertical, horizontal, and skew), followed by 11 secondary collimators (TCSG), and, as a third stage, five movable absorbers (TCLA), either in the vertical or horizontal plane. The two adjustable jaws of primary and secondary collimators are assumed to be made of carbon-fiber composite and to be 60 and $100 \mathrm{~cm}$ long, respectively, and set with a $6.7 \sigma$ $(0.82 \mathrm{~mm})$ and $9.1 \sigma(1.32 \mathrm{~mm})$ half gap [all beam rms sizes $(\sigma)$ cited, refer to a normalized rms emittance of $2.5 \mu \mathrm{m}$ and top energy]. As the entrance and exit of each jaw feature a tapered transition of $10 \mathrm{~cm}$ length, the actual length of the collimator jaw is $20 \mathrm{~cm}$ longer (as seen in Fig. 3). It should be noted that this material choice does not yet account for impedance considerations, although low-impedance materials in the secondary collimators, such as the ones considered for HL-LHC [7], are not expected to significantly change the cleaning efficiency. For the movable absorbers, the material is a tungsten alloy (Inermet180), with a length of $100 \mathrm{~cm}$ and a half gap of $11.5 \sigma(1.04 \mathrm{~mm})$.

Apart from collimators, the cleaning insertion consists of eight warm dogleg magnets (MBW), 24 warm quadrupoles (MQW), and three passive absorbers (TCAP). The $3.4 \mathrm{~m}$ long warm dipoles operate at top energy with a magnetic field of $1.66 \mathrm{~T}$. The MQWs are about $40 \mathrm{~cm}$ longer than in the LHC and provide a gradient of $41 \mathrm{~T} / \mathrm{m}$, while the passive absorbers are the same as in the LHC.

In the collimators closest to the beam (TCP), a fraction of protons, rather than being absorbed and so generating secondary particle showers, undergoes only a limited energy loss in nuclear diffractive processes. Such protons can bypass downstream collimators in the straight section and be lost in the DS where the dispersion increases, giving rise to a global bottleneck in the IR7 DS. In order to address this crucial issue, similarly to what is planned in the context of the HL-LHC, two local DS collimators (TCLD) are foreseen downstream of the LSS to intercept diffracted protons. Those collimators are made of Inermet180 and are assumed to operate with a half gap equal to $18.1 \sigma$ in cell 8 and $22.2 \sigma$ in cell 10. For the HE-LHC, the DS collimators are accommodated by increasing the length of the DS by about $3 \mathrm{~m}$. This gives a geometry offset with respect to the LHC tunnel, which has been investigated and deemed acceptable [28]. A similar solution was considered in the past for the HL-LHC [29] but it was later abandoned in favor of a solution where a main dipole in the DS is replaced by two shorter and stronger magnets (11 versus $8.3 \mathrm{~T}$ ) so as to provide the required space in between [7]. This HL-LHC approach could not be followed for the HELHC, since the availability of stronger magnets, i.e., with a field much higher than the 16 T of the HE-LHC arc dipoles, appears quite uncertain.

\section{SIMULATION WORKFLOW}

As discussed previously, the dedicated insertion for betatron cleaning (IR7) is intended to localize the interception of beam halo particles that would otherwise be lost all along the ring and in particular in the cold arcs. To anticipate and improve the performances of the collimation system in terms of cleaning efficiency as well as induced power deposition, a reliable simulation chain is required. The one followed in the present study, already proven for the LHC $[9,14]$, is recalled in the next subsections.

\section{A. Beam particle tracking around the ring}

To track the halo protons and estimate the loss pattern around the ring and specifically in the collimators, the SixTrack-FLUKA on-line coupling [13] is used. SixTrack tracks the particles along the accelerator magnetic lattice. When these arrive at the collimators, their phase space coordinates are exchanged with FLUKA, which handles their interactions within the jaw material. This tracking procedure is repeated each turn until a particle is lost, namely when the proton undergoes an inelastic interaction, without any generated secondary proton above a certain magnetic rigidity, or if it hits the machine aperture. This method ensures that the effect of the multiturn passage of particles through the collimator is properly taken into consideration. Then, maps of the particles, as they touch the first collimator on any turn, are used as the input for the second step of the simulation chain (see Sec. III B).

Like for the FCC-hh, the vertical halo is deemed more critical than the horizontal in terms of the load on the most impacted collimators, since it hits the most upstream collimator and hence the shower is further developed at the following collimators [12]. The DS losses are on the other hand rather similar. Hence, we focus here on the vertical losses. The initial particle distribution is an annular halo, as defined in [9], with a significant thickness in the vertical plane, distributed in such a way as to hit the vertical primary collimator on the first turn at a very shallow impact depth, and with a normal distribution cut in the horizontal plane, without energy spread. For the results presented here, referring by construction to the vertical halo impacting the most upstream collimator, 95 million particles were tracked for 200 turns, by using the collimator settings mentioned before, for the case of a perfect machine in the $23 \times 90$ lattice version 0.4 .

\section{B. Particle shower calculation}

Secondary particle showers are initiated by the interaction of primary protons with the jaws of directly impacted collimators. In the second simulation step, FLUKA is used to describe the secondary particle propagation into the downstream elements, based on the detailed IR7 geometrical model. This allows to assess the energy deposition in the different elements along the line.

The FLUKA geometry has been assembled using the LineBuilder [30], which is a code reading the sequence and magnetic strengths from the output of the MAD-X 


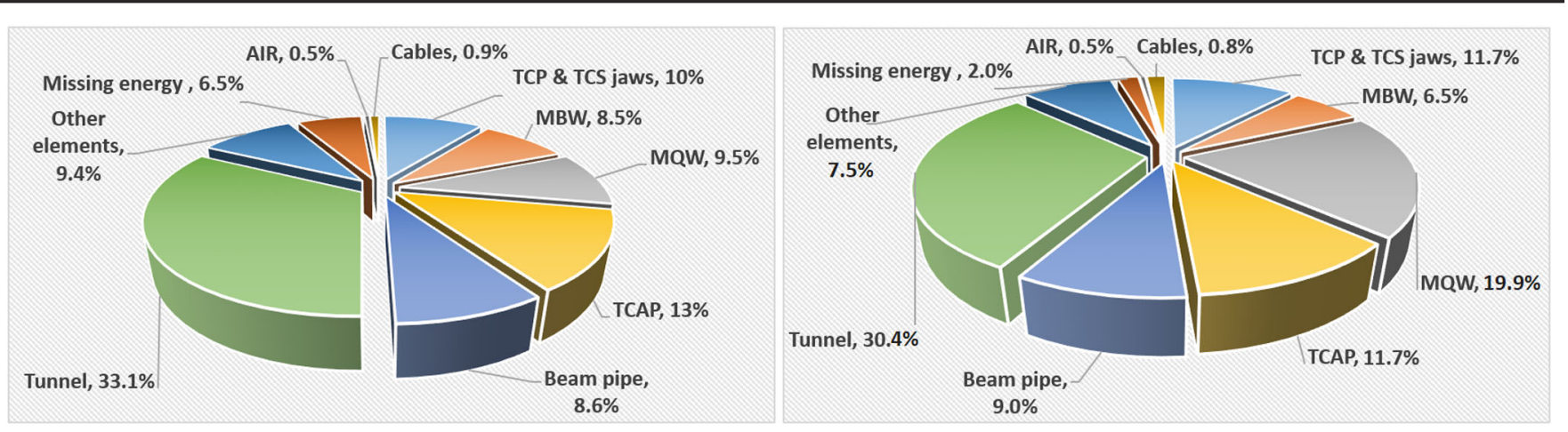

FIG. 2. Power percentages on different LSS elements for LHC at 6.5 TeV (left) and HE-LHC at 13.5 TeV (right) beam energy. Missing energy indicates the energy converted to mass as well as the energy carried away by neutrinos.

program [31]. It places accordingly the beam line element models taken from the FLUKA element database and automatically provided with their specific settings, such as magnetic field and half gap for active magnets and collimators, respectively. A view of the LSS geometry, extending over 500 meters, is shown in Fig. 1.

\section{ENERGY DEPOSITION}

This section reports the thermal loads on different elements and the power density on the collimators, as input for further thermomechanical evaluations, as well as on the SC coils, allowing to quantify the margin to quench. As indicated earlier, these values refer to a total power loss on the collimation system corresponding to $12 \mathrm{~min}$ BLT, for a beam of 2808 bunches with $2.2 \times 10^{11}$ protons per bunch. The peak dose in the warm magnet coils is also given, to determine their lifetime as a function of the annual proton loss amount.

\section{A. IR7 warm section}

We studied beam 1 (B1), which is circulating clockwise in the IR7 external vacuum chamber.

The majority of the betatron halo proton energy is deposited along the LSS. The power percentages on the different geometry elements are shown in Fig. 2, in comparison with the LHC case with data taken from [32]. The largest power fraction is always absorbed by the tunnel walls. The main difference is given by the doubling of the fraction collected by the 24 warm quadrupoles, which one can attribute to their increased length and the higher energy shower onset. Not visible in Fig. 2, a tiny energy fraction at the per mil level turns out to leak into the cold section. The amount of such a diffractive proton leakage in the DS is in percentage rather equivalent for HE-LHC $(0.097 \%)$ and HL-LHC $(0.11 \%)$.

\section{Collimators}

The total power absorbed by the primary and secondary collimators is listed in Table II and the total power on the metallic active absorbers is listed in Table III. The third primary collimator and the first secondary collimator stand out, exceeding $50 \mathrm{~kW}$. Although the integral power on the directly impacted collimator (TCP.D) is relatively low (about $4 \mathrm{~kW}$ ), here one can find the highest local power density. Considering a resolution of $(5 \mu \mathrm{m}, 5 \mu \mathrm{m}, 1 \mathrm{~cm})$ along the $(\mathrm{x}, \mathrm{y}, \mathrm{z})$ axes (pointing outside the ring, opposite to the gravity, and directed as the beam, respectively), on its surface a value of about $40 \mathrm{~kW} / \mathrm{cm}^{3}$ is reached, while the following primary collimators get orders of magnitude less, since they are mostly impacted by the secondary particle

TABLE II. Total power taken by the collimators for $0.2 \mathrm{~h}$ BLT with total loss power of $1.86 \mathrm{MW}$.

\begin{tabular}{lc}
\hline \hline TCP & Power $(\mathrm{kW})$ \\
\hline TCP.D6L & 3.7 \\
TCP.C6L & 29.5 \\
TCP.B6L & 53.1 \\
\hline \hline TCSG & Power $(\mathrm{kW})$ \\
\hline TCSG.A6L & 56.1 \\
TCSG.B5L & 12.6 \\
TCSG.A5L & 37.3 \\
TCSG.D4L & 6.9 \\
TCSG.B4L & 3.7 \\
TCSG.A4L & 4.9 \\
TCSG.A4R & 5.3 \\
TCSG.B5R & 0.5 \\
TCSG.D5R & 1.2 \\
TCSG.E5R & 1.9 \\
TCSG.6R & 0.2 \\
\hline \hline
\end{tabular}

TABLE III. Total power taken by the active absorbers for $0.2 \mathrm{~h}$ BLT with total loss power of $1.86 \mathrm{MW}$.

\begin{tabular}{lc}
\hline \hline TCLA & Power $(\mathrm{kW})$ \\
\hline TCLA.A6R & 3.0 \\
TCLA.B6R & 0.3 \\
TCLA.C6R & 0.1 \\
TCLA.D6R & 0.1 \\
TCLA.A7R & 0.03 \\
\hline \hline
\end{tabular}




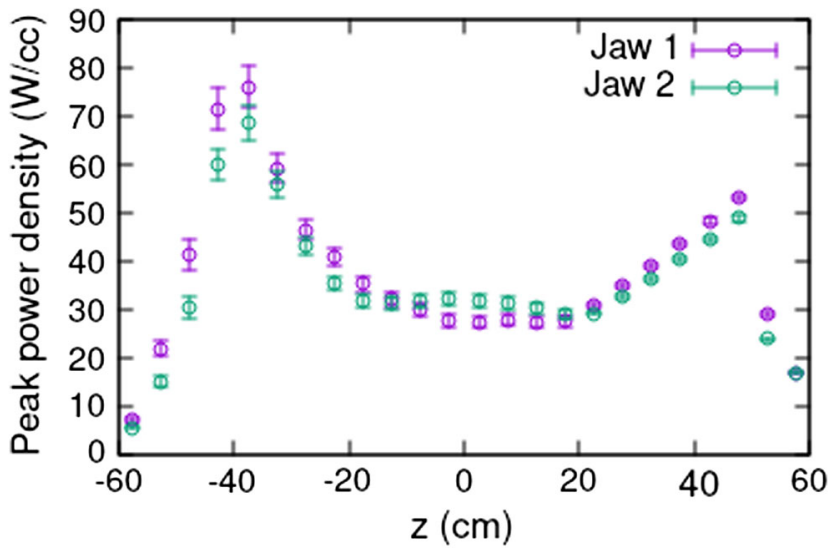

FIG. 3. Peak power density profile on the most exposed secondary collimator for $0.2 \mathrm{~h}$ BLT.

showers developed upstream and not by the halo protons concentrated within a minimal impact parameter range.

For the most exposed secondary collimator (the first one), the peak power density profile is plotted in Fig. 3, where a maximum value of $80 \mathrm{~W} / \mathrm{cm}^{3}$ is reached, which is about a factor 10 higher than in the LHC. The corresponding total power of $56 \mathrm{~kW}$ (see Table II) is about 4 times the respective load in the present machine. However, the three-dimensional distribution of power density (Fig. 4) indicates that the maximum is actually located on the metallic wall of the cooling pipe, rather than on the jaw material. The elevated energy deposition within the high-density material of cooling pipes is due to the impacting secondary halo particles coming from the upstream collimators. A potential concern is thermal deformations, strains, and stresses on the different components, due to the temperature gradient and the thermalexpansion coefficient mismatch between the materials.

As was already shown for the FCC-hh [12,33], a substantial mitigation can be achieved by thickening the collimator jaws by $1-2 \mathrm{~cm}$, and, thereby, keeping the power density maximum inside the latter, at a significantly reduced value thanks to their lower material density. Concerning the

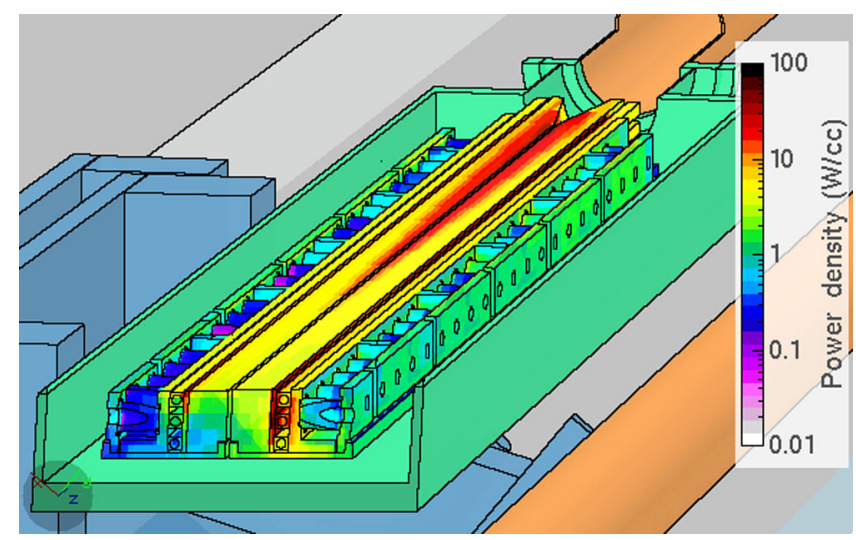

FIG. 4. Power density 3D distribution on the most exposed secondary collimator for $0.2 \mathrm{~h}$ BLT.

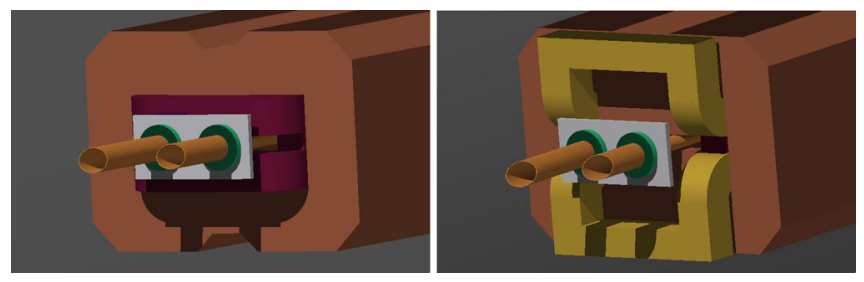

FIG. 5. Geometry model of the warm dipoles in LHC (left) and HE-LHC (right), highlighting the different coil design at the magnet extremity.

final size of the collimator tank and the distance to the second beam pipe - after thickening the jaws - the current available space indicates no issues to arise. In case of FCC-hh, a thermomechanical analysis was performed, and the only permanent deformation was found to occur on the cooling pipes, which can be addressed by adopting a higher yieldstrength material [12]. For the present study with lower power values, therefore, we do not expect any issues concerning the collimator robustness. However, an independent thermomechanical evaluation is required in order to obtain more details, which would be part of any future design studies.

The TCLAs are located far from the directly impacted collimator and feature a large half gap (11.5 sigma), so the total power listed in Table III represents only $0.19 \%$ of the total power loss (1.86 MW for $0.2 \mathrm{~h}$ BLT). In Fig. 2, the corresponding power fraction is included in the "other elements."

\section{Warm dipoles}

Figure 5 highlights a modification [34] we implemented in the MBW model, in order to overcome a well-known weak point of the present LHC warm dipoles, namely the fact that, at the magnet extremities, the coils come close to the beam pipe and are, thereby, exposed to high radiation doses. The problem called for the late installation of dedicated tungsten pieces [32], while we rely here on the adoption of an improved magnet design, keeping the

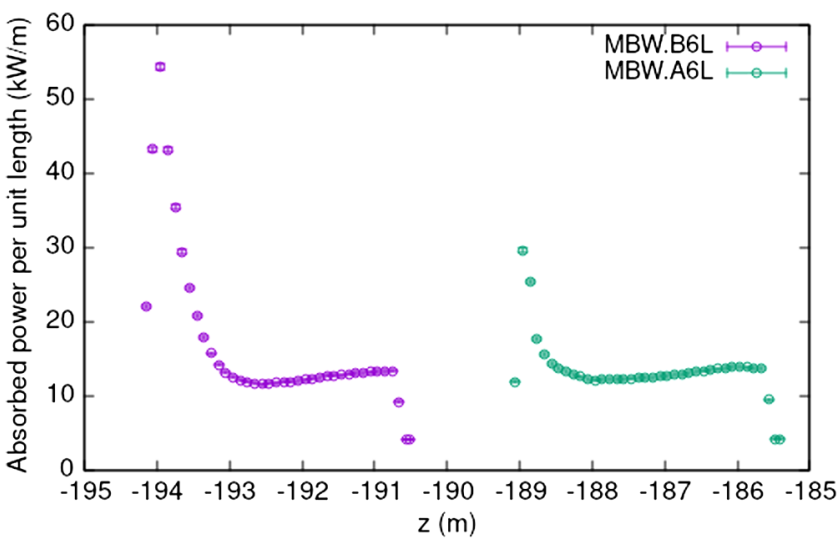

FIG. 6. Absorbed power per unit length in the two most exposed warm dipoles for $0.2 \mathrm{~h}$ BLT. 


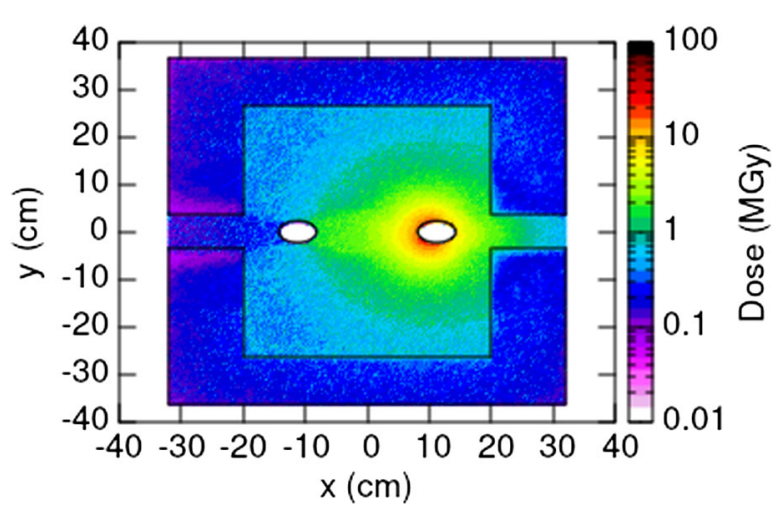

FIG. 7. Dose distribution on the MBW.B6L7 non-IP face for a cumulative loss of $10^{16}$ top energy protons per beam on the collimation system. Values are averaged longitudinally over $7 \mathrm{~cm}$.

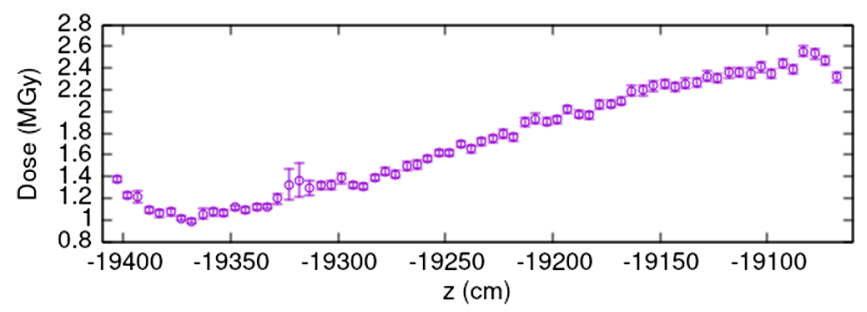

FIG. 8. Longitudinal peak dose profile in the coils along the MBW.B6L7 length for a cumulative loss of $10^{16}$ top energy protons per beam on the collimation system. The dose is evaluated with a resolution of $0.25,0.25$, and $5 \mathrm{~cm}$ in $\mathrm{x}-\mathrm{y}-\mathrm{z}$, respectively.

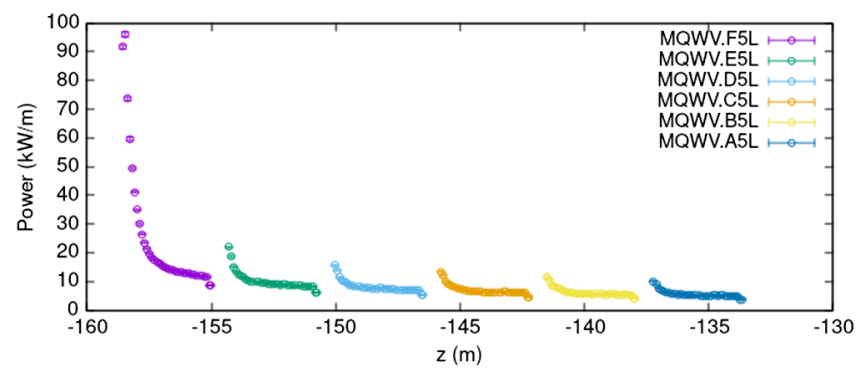

FIG. 9. Absorbed power per unit length in the warm quadrupoles of cell $5 \mathrm{~L}$ for $0.2 \mathrm{~h}$ BLT.

front coils as far as possible from the vacuum chambers, as in the right frame of Fig. 5. The benefit of this measure is quantified below.

Among the eight dipoles enabling the dogleg layout, which further separates the two beam pipes in the middle of the LSS (see Fig. 13), the two magnets downstream of the primary collimators take together more than $99 \%$ of the integral power over all MBWs due to B1 losses, namely 66 and $53 \mathrm{~kW}$, respectively. This is about a factor 3 higher than in the LHC case, which - if critical — can be tackled by designing an adequate shield and/or adapting the cooling system.

Figure 6 shows the absorbed power per unit length, and its longitudinal distribution along these two warm dipoles.
TABLE IV. Total power taken by the warm quadrupoles in cell 5 , on the left side of IP7, for $0.2 \mathrm{~h}$ BLT.

\begin{tabular}{lc}
\hline \hline MQW & Power $(\mathrm{kW})$ \\
\hline MQWV.F5L & 95.1 \\
MQWV.E5L & 39.0 \\
MQWV.D5L & 31.1 \\
MQWV.C5L & 26.8 \\
MQWV.B5L & 23.5 \\
MQWV.A5L & 21.1 \\
\hline \hline
\end{tabular}

The plot features two peaks at 55 and $30 \mathrm{~kW} / \mathrm{m}$, which indicate the accumulation of power at the entrance of either magnet, while for the rest of these magnets the power remains below $14 \mathrm{~kW} / \mathrm{m}$. If needed, the introduction of a suitable front shielding/mask could remove the power peaks. A similar study for FCC-hh demonstrated a reduction of the total power on the magnets by about $10 \%$ [33].

Figure 7 shows the accumulated dose on the front face of the first warm dipole right after the primary collimators, for a reference annual loss amount of $10^{16}$ top energy protons per beam on the collimation system. Thanks to the improved MBW design, where the coils are kept far from the beam pipe also at the magnet extremity, their maximum dose is reduced by 1 order of magnitude.

Figure 8 displays the respective longitudinal peak dose profile in the coils along the magnet. With a maximum dose value of $2.5 \mathrm{MGy}$ per year, a lifetime of at least 10 years is expected, assuming that the coil insulator can withstand about 30 MGy.

\section{Warm quadrupoles}

Figure 9 presents the absorbed power per unit length along the six warm quadrupoles in cell 5 on the left side of IP7, right after the first secondary collimator. The respective total power is indicated in Table IV.

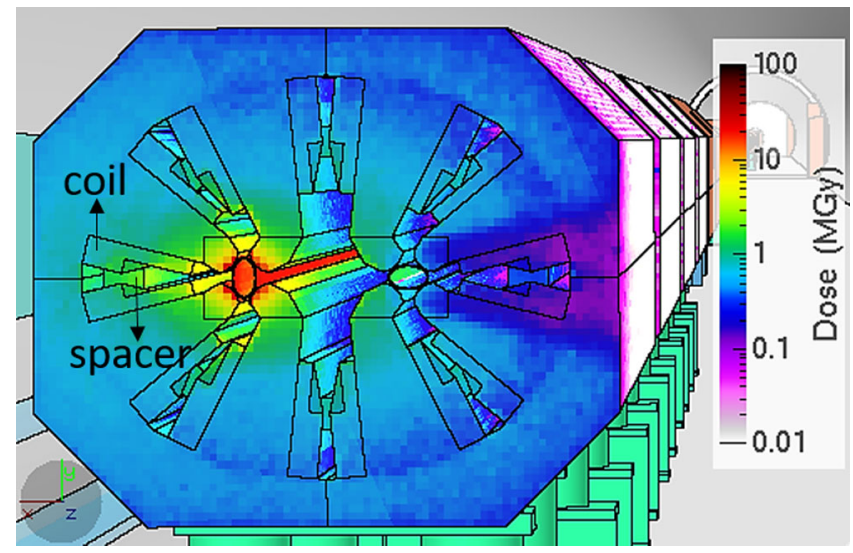

FIG. 10. Dose distribution on the MQWV.F5L for a cumulative loss of $10^{16}$ top energy protons per beam on the collimation system. 

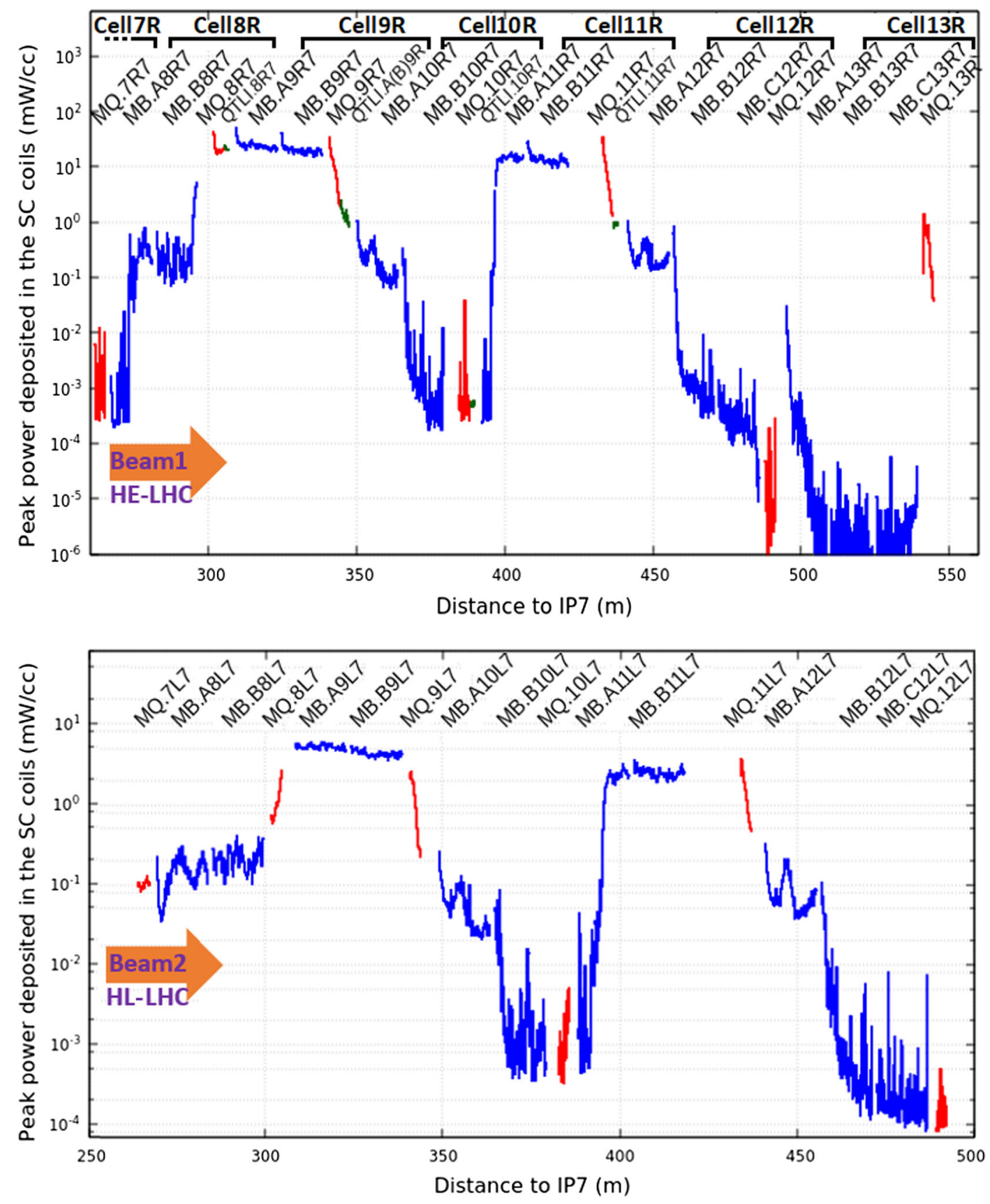

FIG. 11. Profile of peak power density in the SC coils along the IR7 cold magnets of HE-LHC (top) and HL-LHC (bottom) [38]. In the HE case, the TCLD collimators are kept fully open, while the HL case refers to the current layout, where the main dipole replacementmeant to accommodate the TCLD collimator-did not take place yet. Blue corresponds to the main dipoles, red to the main quadrupoles, and dark green to the trim quadrupoles. Values are calculated for $0.2 \mathrm{~h}$ BLT and are radially averaged over the SC cable thickness.

Despite the passive absorber (TCAP—shown in Figs. 1 and 13) introduced ahead of the first MQW (MQWV.F5L) in order to protect it against the particle shower coming from the upstream collimators, this remains the most loaded magnet among all the IR7 warm magnets. By design of a suitable cooling system, the warm magnets are able to dissipate such level of linear power densities - as already tested at CERN [35].
Figure 10 presents a pictorial view of its annual dose distribution. The maximum dose value in the coils is about $4 \mathrm{MGy}$, which, for the expected material limit of $50 \mathrm{MGy}$, translates into a lifetime of about 10 years, which would correspond to about half of the total HE-LHC operation time envisaged [3]. Compounding the problem, the plastic spacer used to hold the four separate coils is less radiation resistant and could be critically damaged already after 
exposure to $10 \mathrm{MGy}[36,37]$. With this consideration, the resulting dose at the spacers, even though lower than in the coils, is still excessive. Nevertheless, we are confident that an effective mitigation can be achieved by following the same approach as for the HL-LHC, where both a front tungsten mask and internal shielding pieces placed between the beam pipe and the coils are implemented [37].

\section{B. IR7 cold section}

In addition to shorter corrector magnets, the DS cold section hosts the main quadrupoles (MQ) of $3.5 \mathrm{~m}$ magnetic length and $347 \mathrm{~T} / \mathrm{m}$ gradient, the main bending dipoles (MB) of $13.83 \mathrm{~m}$ magnetic length and $16.6 \mathrm{~T}$ field, and the trim quadrupoles of $1.3 \mathrm{~m}$ length. The DS layout can be deduced from the top plot in Fig. 11.

As only a small fraction of protons (at the per mil level) is expected to leak into the cold section, unaffordable CPU times would be required to obtain enough statistics for a representative shower simulation and reliable power calculation in the DS. In order to overcome this challenge, an extra step in the simulation chain is adopted by means of FLUKA, where only very high-energy particles are tracked through the LSS and then their information is stored when hitting the cold section's aperture. The map of these particles, mostly protons, is then used as the input for the final step, to simulate locally the induced shower development and to calculate the power deposition in the DS elements. This method omits the additional load that the very first magnets (namely the main quadrupole in cell 7, the first dipole and the initial part of the second dipole in cell 8) are subject to, due to the lower energy particle shower that leaks from the LSS but which is unable to reach cell 9. This contribution was independently evaluated and found not to affect the identification of the DS hot spot, which in any case lies downstream. It is explicitly quantified in Sec. V, where the radiation exposure of the MB.A8R7 dipole is investigated for other purposes.

In the present study, we have evaluated scenarios both with and without the two TCLDs in the cold section to intercept the out-scattered off-momentum protons (one at the end of cell 8, before MQ8, and the other right before MQ10). The results presented in the following clearly demonstrate the need for the TCLDs, which are also integrated in the HE-LHC baseline.

Figure 11 reports the longitudinal peak power profile along the SC coils in the DS magnets, without TCLD collimators. For comparison purposes, the HL-LHC picture is also shown [38]. The latter refers to beam 2, contrary to our present HE-LHC case where beam 1 is studied. Nevertheless, the layout and optics are almost fully symmetric and the results can thus be compared. The maximum value is found to be on the first SC dipole of cell 9 and is equal to 7 and $25 \mathrm{~mW} / \mathrm{cm}^{3}$ in the HL-LHC and HE-LHC, respectively (for $0.2 \mathrm{~h}$ BLT). In both cases, it is due to overbent protons hitting the inner side of the beam pipe. The factor of 3.5 rise reflects both the proton loss increase and the beam energy doubling for HE-LHC.

Figure 12 shows that the addition of the DS collimators, operating with a half gap of about $20 \sigma$, as specified earlier, changes the pattern. It makes the preceding dipole (i.e., the second in cell 8) the most exposed one and, thereby, yields a considerable gain in the maximum power density, which becomes about 5 times smaller.

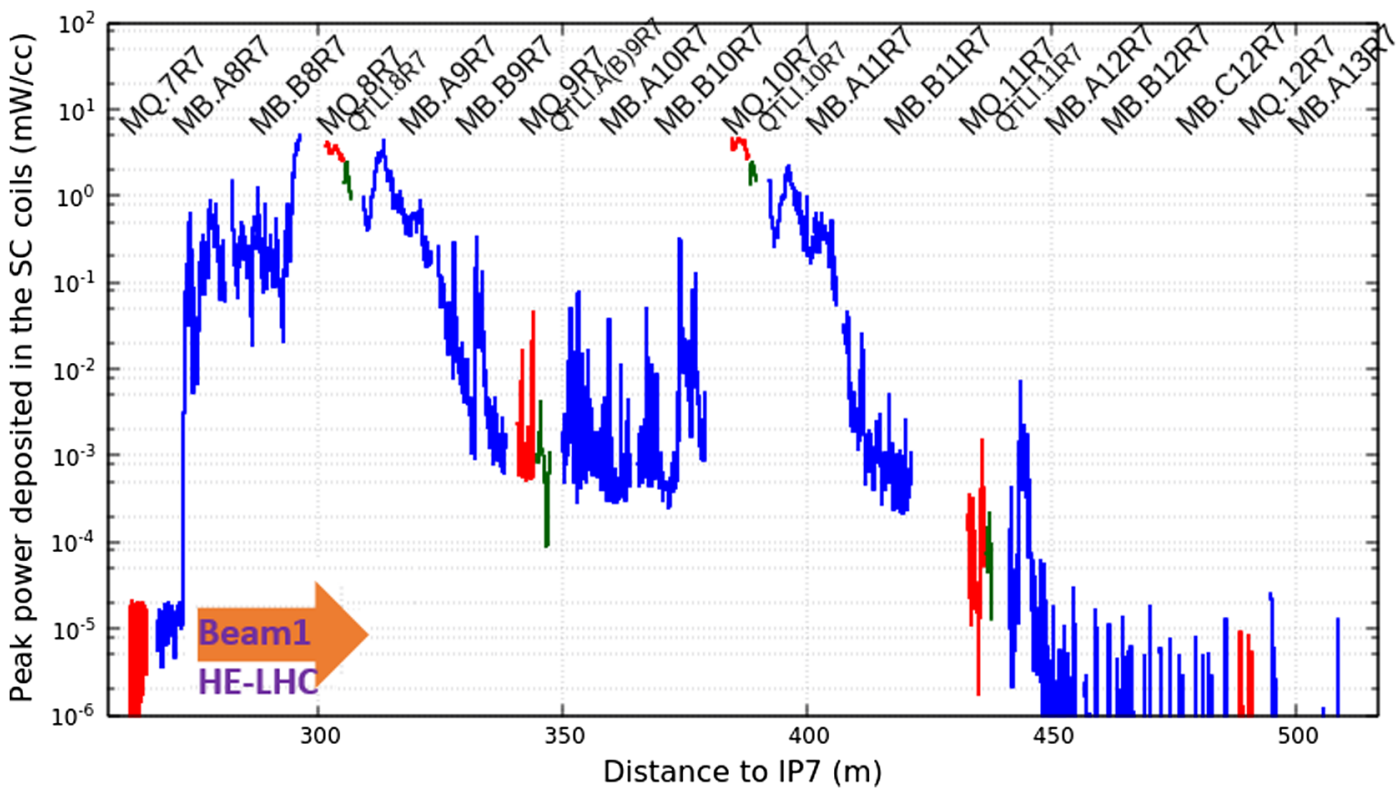

FIG. 12. Profile of peak power density in the SC coils along the IR7 cold magnets of HE-LHC, in the presence of TCLD collimators (one at the end of cell 8, before MQ8, and the other at the end of cell 10 just before MQ10). The color code is as in Fig. 11. Values are calculated for $0.2 \mathrm{~h}$ BLT and are radially averaged over the SC cable thickness. 


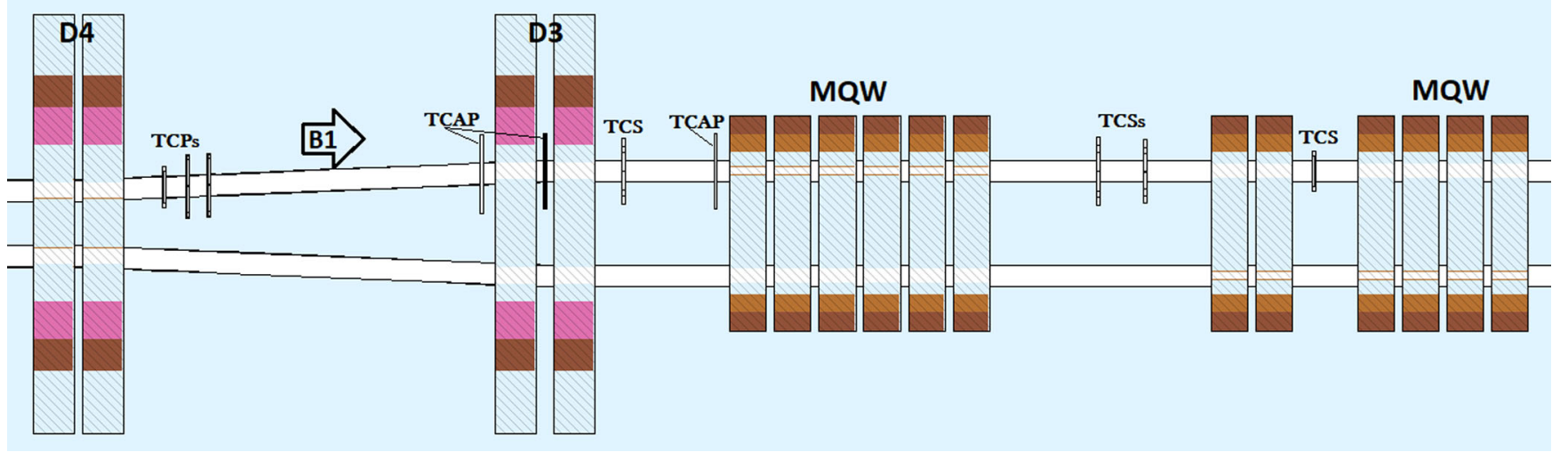

FIG. 13. Portion of the FLUKA model of the betatron collimation insertion, including warm dipoles (D3 and D4), collimators (TCP and TCS), warm quadrupoles (MQW), and passive absorbers (TCAP). The increase of the separation between the two beam pipes (from 204 to $224 \mathrm{~mm}$, with reference to their axes) defines the so-called dogleg.

It shall be noted that the LHC experience indicates an underestimation of the DS losses when simulating an ideal machine. Measurements suggest that the number of protons leaking into the DS is a factor of 3 higher than predicted, which may be due to machine imperfections, such as misalignment of the collimator jaws [15]. Previous studies have shown that combined imperfections can indeed increase the DS peak losses by this amount [9], although it remains as future work to redo a similar imperfection study for the HE-LHC.

One should also distinguish between power density averaged over the radial cable thickness (around 15$20 \mathrm{~mm}$, as considered so far in the current paper section) and the local power density on the cable inner edge (over an interval of a few $\mathrm{mm}$, for which, at the HE-LHC proton energy, the power-density value is up to 2-3 times higher). With a local quench limit in the 70 to $100 \mathrm{~mW} / \mathrm{cm}^{3}$ interval for the $16 \mathrm{~T}$ dipole, the maximum value of $5 \mathrm{~mW} / \mathrm{cm}^{3}$ with TCLDs in Fig. 12 offers a good margin even after multiplying it both by the above underestimation factor (3) and the local to averaged power density factor $(2-3)$. On the other hand, for the case without TCLDs, the peak of $25 \mathrm{~mW} / \mathrm{cm}^{3}$ would, with the two factors included, clearly exceed the quench limit, which demonstrates the necessity of the TCLDs.

\section{ROLE OF THE DOGLEG}

As previously pointed out, the LSS warm dipoles are used to increase the beam pipe separation in the middle of the insertion (amounting to $224 \mathrm{~mm}$ between the two pipe axes) with respect to the one in the arc (204 $\mathrm{mm})$, as shown in Fig. 13, to provide room for TCSGs. The warm dipoles are subject to a very high power load. To avoid this challenging situation, an alternative could be to not use such a dogleg, which might be feasible for HE-LHC as sufficient transverse space is available in the LSS. In this section of the paper, we discuss the effect of the removal of the dogleg, by comparing the baseline layout illustrated in the figure with an alternative layout that maintains the same beam separation as in the arc (but still includes the eight dipoles for the sake of comparison-if not used, they could be replaced by equivalent shielding). The evaluation is performed in terms of the respective radiation impact on the first SC main dipole at the beginning of the DS, where the beam lines start to bend after the LSS.

The halo protons first impact one of the primary collimators (the vertical one in the studied loss scenario) sitting between the D4 and D3 warm dipole pairs. As a result of their nuclear reactions in the collimator jaws, highly energetic secondary particles emerge along the beam direction. The neutral ones, mostly photons (particularly abundant) and neutrons, proceed straight in the vacuum chamber, as they are insensitive to the magnet field. In the absence of the dogleg, they have a considerable chance to reach the end of the straight section without being intercepted and to collide against the bent aperture of the first dipole in cell 8, as sketched in Fig. 14. This situation is quantified in Table V, where the yield of photons and neutrons hitting the stainless steel beam screen of the MB.A8R7 magnet is given for the two considered layouts, highlighting a major difference. The latter is further emphasized when looking at the respective energy spectrum, which for neutrons approaches the beam energy

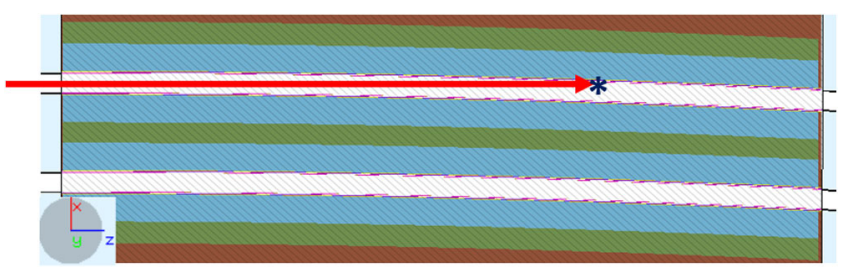

FIG. 14. Schematic trajectory of high-energy neutral particles coming straight from the LSS and crossing, at the indicated point, the boundary of the B1 mechanical aperture of the first DS main dipole. 
TABLE V. Rate of neutral particles hitting the first main dipole aperture, with and without the dogleg.

\begin{tabular}{lcc}
\hline \hline & Number of particles per lost proton \\
\hline Type & Without dogleg & Nominal dogleg \\
\hline Photons & $4 \times 10^{-3}$ & $4 \times 10^{-5}$ \\
Neutrons & $3 \times 10^{-4}$ & $<4 \times 10^{-7}$ \\
\hline \hline
\end{tabular}

without the dogleg, while it does not exceed $2 \mathrm{TeV}$ in the other case.

Moreover, with respect to the case with nominal dogleg, a larger number of charged pions-also originated by the inelastic interactions of the halo protons with the jawsleave the LSS and are captured by the high magnetic field of the first main dipole in the DS. Thanks to the design of the nominal dogleg, the angle given to the primary collimators directs the above-mentioned neutral and charged particles outside of the main ring in the arc.

Also for this study, the FLUKA calculation consisted of two steps. In the first step, the source term was represented by the halo protons entering the collimator jaws, obtained as explained in Sec. III A, but this time the simulation also followed intermediate energy particles, in order to properly characterize the relevant radiation field on a virtual plane located at $25 \mathrm{~m}$ from the LSS end. The resulting map of particles was then used as the input for the last step, where the full shower propagation into the first segment of the cold section was simulated in order to calculate the energy deposition on the first SC magnets. Here, we also took into account the additional LSS leakage, which we had neglected in Sec. IV B.

Figure 15 accounts for the SC coil exposure of the first dipole in cell 8. In the absence of the dogleg, two marked

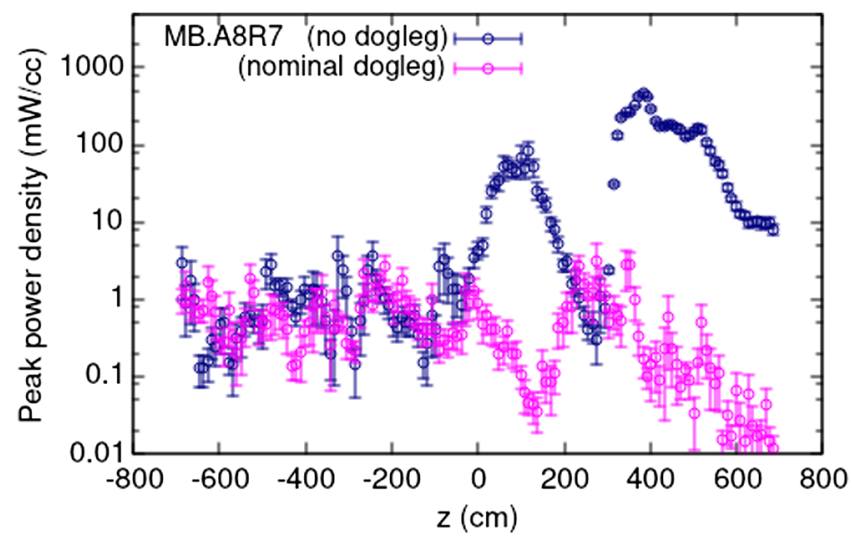

FIG. 15. Peak power density profile in the SC coils of the first main bending dipole in the DS (MB.A8R7), with and without the dogleg. Values are calculated for $0.2 \mathrm{~h}$ BLT and are radially averaged over the SC cable thickness. For the nominal dogleg case, they are higher than in Figs. 11 and 12 due to the additional shower contribution discussed in the text. Vertical bars, where visible, indicate the statistical uncertainty. peaks arise. One is produced by the impact of a large number of charged pions and the other, exceeding $500 \mathrm{~mW} / \mathrm{cm}^{3}$ (for $0.2 \mathrm{~h} \mathrm{BLT}$ ) at $3 \mathrm{~m}$ from the magnet's end, is caused by the localized collision of neutral particles on the external wall (with respect to the ring center) of the beam screen. Moreover, the total power collected by the $\sim 14 \mathrm{~m}$ magnet is 30 times higher than for the baseline layout featuring the dogleg. This comparison proves the dogleg to be essential from the machine protection point of view. On the other hand, a further increase of the beam separation did not lead to any noticeable additional improvement.

\section{CONCLUSIONS}

We have evaluated the radiation impact on the accelerator elements in the betatron cleaning insertion of the proposed future high-energy LHC, considering a conservative regular beam loss scenario. That is, we assumed a vertical beam halo impacting on the collimation system at a rate corresponding to a beam lifetime as low as $0.2 \mathrm{~h}$, thereby applying the design criterion that was adopted for the LHC. This is a highly challenging scenario, which, for the HE-LHC, results in a total power load of $1.86 \mathrm{MW}$ of the lost protons, given the stored beam energy of 1.34 GJ. Relying on the LHC concept for the layout and optics of the collimation systemcomplemented by collimators in the dispersion suppressors, similar to those planned for the high luminosity LHC, but much more difficult to implement optics-wise [3,28] — no showstopper has arisen, despite the higher beam energy and intensity. This is a very important conclusion for future design studies of the HE-LHC.

Nevertheless, for a few heavily loaded collimators, thicker jaws shall be envisaged, in order not to directly expose their cooling pipes to the electromagnetic shower development. Moreover, the primary collimator intercepting the beam halo will experience a challenging power density on its jaw surface. Fortunately, thermomechanical studies performed for the case of the Future Circular hadron Collider (FCC-hh), where the conditions are even more severe, indicate that the consequences can be suitably addressed [12].

The three passive absorbers in front of the most impacted warm magnets do not provide a sufficiently effective protection in the HE-LHC machine, calling for a more massive design to significantly reduce the power deposition over the first meter of the magnet concerned. The path of the magnet coils at the warm dipole extremity shall be optimized so as to keep them far from the beam pipe, contrary to the present LHC magnet, in order not to excessively shorten their lifetime. The plastic type used for the quadrupole coil spacers should ideally be replaced by a more resistant material, or be protected by inner metallic shields as later implemented in the LHC.

Importantly, the inclusion of two collimators in the dispersion suppressors, intended to intercept diffractive protons otherwise hitting the cold magnet aperture, was 
shown to be crucial for bringing the quench risk to an acceptable level.

Our simulations have also revealed that, even if not required for integration reasons (e.g., to better accommodate beam line elements such as the collimator tanks), the beam pipe separation enabled by the dogleg layout is essential. Namely, without the dogleg, the first superconducting main dipole after the end of the long straight section would experience a dramatic increase of its power load, mostly due to highly energetic neutral particles generated in the collimator jaws. The installation of the primary collimators in the skew segment of the dogleg line, i.e., between the warm dipole pairs, was shown to prevent such a major threat.

\section{ACKNOWLEDGMENTS}

The authors would like to thank A. Mereghetti, J. Molson, S. Redaelli, and the FLUKA team of the CERNBMI section for all the help provided during this study. This work was supported, in part, by the European Commission under the HORIZON2020 Integrating Activity project ARIES, Grant Agreement No. 73087.

[1] A. Abada et al., FCC-ee: The Lepton Collider, Eur. Phys. J. Special Topics 228, 261 (2019).

[2] A. Abada et al., FCC-hh: The Hadron Collider, Eur. Phys. J. Special Topics 228, 755 (2019).

[3] A. Abada et al., HE-LHC: The High-Energy Large Hadron Collider, Eur. Phys. J. Special Topics 228, 1109 (2019).

[4] A. Abada et al., FCC physics opportunities, Eur. Phys. J. Special Topics 79, 474 (2019).

[5] LHC design report v.1: The LHC main ring, edited by O. S. Brüning, P. Collier, P. Lebrun, S. Myers, R. Ostojic, J. Poole, and P. Proudlock, Report No. CERN-2004-003-V1, 2004, http://cds.cern.ch/record/782076?ln=en.

[6] R. W. Assmann, Preliminary beam-based specifications for the LHC collimators, Report No. LHC-PROJECT-NOTE277, 2002, http://cds.cern.ch/record/691766.

[7] High-luminosity Large Hadron Collider (HL-LHC): Technical Design Report V. 0.1, edited by G. Apollinari, I. B. Alonso, O. Bruning, P. Fessia, M. Lamont, L. Rossi, and L. Tavian, CERN Yellow Reports: Monographs, Report No. CERN-2017-007-M, 2017, https://doi.org/10.23731/ CYRM-2017-004.

[8] B. Salvachua, Overview of proton-proton physics during run 2, in Proceedings of the 9th LHC Operations Evian Workshop, Evian, France, 2019, https://cds.cern.ch/ record/2750272? In=en.

[9] R. Bruce et al., Simulations and measurements of beam loss patterns at the CERN Large Hadron Collider, Phys. Rev. ST Accel. Beams 17, 081004 (2014).

[10] A. Lechner, B. Auchmann, R. Bruce, F. Cerutti, P. P. Granieri, A. Marsili, S. Redaelli, N. V. Shetty, E. Skordis, G. E. Steele, and A. P. Verwei, Power deposition in LHC magnets with and without dispersion suppressor collimators downstream of the betatron cleaning insertion, in
Proceedings of the International Particle Accelerator Conference 2014, Dresden, Germany, 2014, Vol. 17, p. 112, https://doi.org/10.18429/JACoW-IPAC2014-MOPRO021.

[11] R. Bruce, A. Marsili, and S. Redaelli, Cleaning performance with 11T dipoles and local dispersion suppressor collimation at the LHC, in Proceedings of the International Particle Accelerator Conference 2014, Dresden, Germany, 2014, Vol. 17, p. 170, https://doi.org/10.18429/JACoWIPAC2014-MOPRO042.

[12] R. Bruce, A. Abramov, A. Bertarelli, M. I. Besana, F. Carra, F. Cerutti, A. Faus-Golfe, M. Fiascaris, G. Gobbi, A. Krainer, A. Lechner, A. Mereghetti, D. Mirarchi, J. Molson, M. Pasquali, S. Redaelli, D. Schulte, M. Serluca, E. Skordis, and M. Varasteh Anvar, Collimation system studies for the FCC-hh, J. Phys. Conf. Ser. 1350, 012009 (2019).

[13] A. Mereghetti et al., SixTrack-FLUKA active coupling for the upgrade of the SPS scrapers, in Proceedings of the International Particle Accelerator Conference 2013, Shanghai, China, 2013, p. 2657, http://accelconf.web .cern.ch/AccelConf/IPAC2013/papers/wepea064.pdf.

[14] E. Skordis, R. Bruce, F. Cerutti, A. Ferrari, P. D. Hermes, A. Lechner, A. Mereghetti, S. Redaelli, B. M. Salvachua, V. Vlachoudis, and C. P. Welsch, Study of the 2015 top energy LHC collimation quench tests through an advanced simulation chain, in Proceedings of the International Particle Accelerator Conference, Copenhagen, Denmark, 2017, http://accelconf.web.cern.ch/AccelConf/ipac2017/ papers/mopab012.pdf.

[15] A. Lechner, B. Auchmann, T. Baer, C. B. Castro, R. Bruce, F. Cerutti, L. S. Esposito, A. Ferrari, J. M. Jowett, A. Mereghetti, F. Pietropaolo, S. Redaelli, B. Salvachua, M. Sapinski, M. Schaumann, N. V. Shetty, V. Vlachoudis, and E. Skordis, Validation of energy deposition simulations for proton and heavy ion losses in the CERN Large Hadron Collider, Phys. Rev. Accel. Beams 22, 071003 (2019).

[16] R. Bruce, M. Huhtinen, A. Manousos, F. Cerutti, L. Esposito, R. Kwee-Hinzmann, A. Lechner, A. Mereghetti, D. Mirarchi, S. Redaelli, and B. Salvachua, Collimationinduced experimental background studies at the CERN Large Hadron Collider, Phys. Rev. Accel. Beams 22, 021004 (2019).

[17] SixTrack web site, http://sixtrack.web.cern.ch/SixTrack/.

[18] G. Robert-Demolaize, R. Assmann, S. Redaelli, and F. Schmidt, A new version of SixTrack with collimation and aperture interface, in Proceedings of the 21st Particle Accelerator Conference, Knoxville, TN, 2005 (IEEE, Piscataway, NJ, 2005), p. 4084.

[19] G. Battistoni et al., Overview of the FLUKA code, Annals nuclear energy, in Proceedings of the Joint International Conference on Supercomputing in Nuclear Applications + Monte Carlo (SNA+MC 2013): Paris, France, 2013, Vol. 82, pp. 10-18, https://doi.org/10.1016/j.anucene.2014 .11 .007 .

[20] T. T. Bohlen, F. Cerutti, M. P. W. Chin, A. Fassò, A. Ferrari, P. G. Ortega, A. Mairani, P. R. Sala, G. Smirnov, and V. Vlachoudis, The FLUKA code: Developments and challenges for high energy and medical applications, Nucl. Data Sheets 120, 211 (2014). 
[21] V. Vlachoudis, FLAIR: A powerful but user friendly graphical interface for FLUKA, in Proceedings of the International Conference on Mathematics, Computational Methods and Reactor Physics (MC 2009), Saratoga Springs, New York, 2009, http://www.fluka.org/flair/.

[22] R. W. Assmann, Collimators and beam absorbers for cleaning and machine protection, in Proceedings of the LHC Project Workshop-Chamonix XIV, Chamonix, France, 2005, p. 261, https://cds.cern.ch/record/987838.

[23] R. W. Assmann et al., The final collimation system for the LHC, in Proceedings of the 10th European Particle Accelerator Conference, Edinburgh, Scotland, 2006 (EPS-AG, Edinburgh, Scotland, 2006), p. 986.

[24] R. Bruce, R. W. Assmann, and S. Redaelli, Calculations of safe collimator settings and $\beta^{*}$ at the CERN Large Hadron Collider, Phys. Rev. ST Accel. Beams 18, 061001 (2015).

[25] G. Valentino, G. Baud, R. Bruce, M. Gasior, A. Mereghetti, D. Mirarchi, J. Olexa, S. Redaelli, S. Salvachua, A. Valloni, and J. Wenninger, Final implementation, commissioning, and performance of embedded collimator beam position monitors in the Large Hadron Collider, Phys. Rev. Accel. Beams 20, 081002 (2017).

[26] R. Bruce, C. Bracco, R. De Maria, M. Giovannozzi, A. Mereghetti, D. Mirarchi, S. Redaelli, E. Quaranta, and B. Salvachua, Reaching record-low $\beta^{*}$ at the CERN Large Hadron Collider using a novel scheme of collimator settings and optics, Nucl. Instrum. Methods Phys. Res., Sect. A 848, 19 (2017).

[27] D. Mirarchi, R. B. Appleby, A. Bertarelli, R. Bruce, F. Cerutti, H. G. Morales, P. D. Hermes, R. Kwee-Hinzmann, A. Lechner, A. Mereghetti, E. Quaranta, and S. Redaelli, Cleaning performance of the collimation system of the high luminosity Large Hadron Collider, in Proceedings of the International Particle Accelerator Conference 2016, Busan, Korea, 2016, http://accelconf.web.cern.ch/ AccelConf/ipac2016/papers/wepmw030.pdf.

[28] J. Keintzel, R. Tomas, R. Bruce, M. Giovannozzi, T. Risselada, and F. Zimmermann, Lattice and optics options for possible energy upgrades of the Large Hadron Collider, Phys. Rev. Accel. Beams 23, 101602 (2020).

[29] R. Aßmann, G. Bellodi, J. M. Jowett, E. Métral, T. Weiler, L. Keller, and T. Markiewicz, Accelerator physics concept for upgraded LHC collimation performance, in Proceedings of the 23rd Particle Accelerator Conference, Vancouver, Canada, 2009 (IEEE, Piscataway, NJ, 2009).

[30] A. Mereghetti, V. Boccone, F. Cerutti, R. Versaci, and V. Vlachoudis, The FLUKA LineBuilder and element database: Tools for building complex models of accelerators beam lines, in Proceedings of the 3rd International Particle Accelerator Conference, New Orleans, LA, 2012 (IEEE, Piscataway, NJ, 2012), pp. 2687.

[31] MAD-X program, http://cern.ch/mad/.

[32] E. Skordis, R. Bruce, F. Cerutti, A. Ferrari, P. D. Hermes, A. Lechner, A. Mereghetti, P. G. Ortega, S. Redaelli, and V. Vlachoudis, Impact of beam losses in the LHC collimation regions, in Proceedings of the International Particle Accelerator Conference 2015, Richmond, VA, 2015, http://accelconf.web.cern.ch/AccelConf/IPAC2015/papers/ tupty046.pdf.

[33] M. Varasteh Anvar et al., Energy deposition in the FCC-hh betatron cleaning insertion, International Future Circular Collider (FCC) Conference, 2018, https://indico.cern.ch/ event/656491/contributions/2930758/.

[34] A. Milanese (private communication).

[35] D. Tommasini (private communication).

[36] F. Cerutti et al., Radiation levels of MBW and MQW, at the 14th HL-LHC TCC meeting, 2016, https://indico.cern.ch/ event $/ 559125 /$.

[37] C. B. Castro et al., Improved protection of the warm magnets of the LHC betatron cleaning insertion, in Proceedings of the International Particle Accelerator Conference (IPAC'17), Copenhagen, Denmark, 2017, pp. 72-75, https://doi.org/ 10.18429/JACoW-IPAC2017-MOPAB004.

[38] C. B. Castro et al., Energy deposition from collimation losses in the DS region at P7, at the 8th $H L-L H C$ Annual Meeting, 2018, https://indico.cern.ch/event/ 742082/contributions/3085132/. 